\title{
ORIGINAL ARTICLE ASSOCIATION OF ST ELEVATION IN LEAD AVR WITH LEFT MAIN STEM AND TRIPLE VESSEL DISEASES IN PATIENTS WITH NON-ST ELEVATION MYOCARDIAL INFARCTION
}

\author{
Hafiz Tahir Usman ${ }^{1}$, Kashif Ali Hashmi ${ }^{1}$, Muhammad Sohail Saleemi ${ }^{1}$, Ammar Akhtar ${ }^{1}$ \\ ${ }^{1}$ Ch. Pervaiz Elahi Institute of Cardiology (CPEIC), Multan, Pakistan
}

\begin{abstract}
Objectives: To determine frequency of left main stem (LMS) and triple vessel coronary artery disease (3VCAD) in patients of Non-ST-elevation myocardial infarction (NSTEMI) and to compare the frequency of LMS and 3VCAD in patients with NSTEMI with or without ST elevation in lead aVR.

Methodology: Total 346 patients with NSTEMI having age 30-70 years were included in this descriptive cross-sectional study. The data on demographic details was collected. All patients underwent electrocardiography (ECG) and cardiac specific troponin-I assessment. Patients were categorized as NSTEMI with or without ST-elevation in lead aVR. Coronary angiography was performed in all patients and angiographic findings were noted.

Results: Mean age of patients was 51.87 \pm 10.03 years. There were $218(63.01 \%)$ males and 128 (36.99\%) female patients. $182(52.60 \%)$ patients of NSTEMI had ST elevation in aVR. LMS disease was found in $53(29.10 \%)$ patients with ST elevation in aVR. Sensitivity, specificity, positive predive value and negative predictive value of ST elevation in aVR for LMS disease was $62.35 \%, 50.57 \%, 29.12 \%$ and $80.49 \%$ respectively. 3 VCAD was found in $54(29.70 \%)$ with ST elevation in aVR. Sensitivity, specificity, positive predive value and negative predictive value of ST elevation in aVR for 3VCAD was $77.14 \%, 53.52 \%, 29.67 \%$ and $90.24 \%$ respectively.

Conclusion: NSTEMI patients with ST elevation in aVR may have higher chances of having LMS disease or 3VCAD. There is high negative predictive value for ST elevation in aVR to predict LMS disease or 3VCAD.
\end{abstract}

Keywords: NSTEMI, aVR, ST-elevation, Left main stem disease, triple vessel disease

Citation: Usman HT, Hashmi KA, Saleemi MS, Akhtar A. Association of ST Elevation in Lead aVR with Left Main Stem and Triple Vessel Diseases in Patients with Non-ST Elevation Myocardial Infarction. Pak Heart J. 2021;54(04):348-351. DOI: https://doi.org/10.47144/phj.v54i4.2128

\section{INTRODUCTION}

The patients with Non-ST elevation myocardial infarction (NSTEMI) have variable degree of severity of coronary artery disease and prognosis. ${ }^{1}$ The electrocardiogram (ECG) at first medical contact and at the time of admission has a crucial contribution in easy and an early risk stratification. ${ }^{1,2}$ Previous studies have proved that patients with even ST depression of $>0.5 \mathrm{~mm}$ on the admission ECG may need early invasive management strategy. ${ }^{2}$ Different risk scoring methods like TIMI scoring system for NSTEMI have also used ST deviation as an independent predictor of adverse outcome. ${ }^{3,4}$

The lead aVR is often ignored in clinical practice. ${ }^{5}$ However, different studies have shown that ST elevation in lead aVR can predict adverse events in NSTEMI patients more accurately. ${ }^{6,7}$ Furthermore, ST elevation in aVR is a simple and an easy indicator of left main stem (LMS) or triple vessel disease (3VCAD) and can guide for the triage of these highrisk patients. ${ }^{6,8}$ Yan AT et al. showed that among 5064 patients with NSTEMI 5.8\% had minor (0.5-1 mm) ST elevation in aVR, and $1.5 \%$ had major $(>1 \mathrm{~mm}) \mathrm{ST}$ elevation in aVR. Triple vessel disease was found in $46.5 \%$ patients with ST elevation in aVR compared to $26.1 \%$ with no ST-elevation in aVR. ${ }^{9}$

Considering the literature, the rationale of our study is to find out the diagnostic value of ST elevation in aVR with LMS disease or 3VCAD for the patients with NSTEMI. These patients will be further investigated with coronary angiography. If lead aVR will have diagnostic value of $80 \%$ for LMS disease or 3VCAD, then in future this prognostic information from ECG could be taken routinely to diagnose LMS disease or 3VCAD.

It will help in early anticipation of LMS and 3VCAD on the basis of ECG of the patient presenting with NSTEMI. This will also help to make local guidelines regarding early prediction of disease, proceed for angiography and patient's definitive management. 


\section{METHODOLOGY}

This descriptive cross-sectional study was carried out in the department of Cardiology at Chaudhary Pervaiz Elahi Institute of Cardiology, Multan from 1st of November, 2020 to 30th of April 2021. After approval from the Ethical Review Board of the hospital and taking informed consent from the patients, a total 346 consecutive patients with NSTEMI of either gender of age 30 to 70 years were included in the study. Patients with previous history of acute coronary syndrome, coronary angiography, coronary revascularization procedure, nephropathy, severe left ventricular failure or cardiogenic shock were not included in the study. NSTEMI was diagnosed by the history of severe excruciating or crushing chest pain with radiation to left arm, neck jaw or back and a positive cardiac troponin I marker with either ST depression in any 2 of the consecutive leads. On 12-lead ECG, ST elevation of more than $0.5 \mathrm{~mm}$ in lead aVR was labelled as ST-elevation in aVR. Left main stem (LMS) was defined as significant narrowing (more than $50 \%$ ) of the left main coronary artery on coronary angiogram. Triple vessel disease was labelled as significant narrowing $(>75 \%)$ of all three major coronary arteries on coronary angiogram. The patients were labeled diabetic if already using hypoglycemic drugs either oral or parenteral or fasting blood sugar level of more than $126 \mathrm{mg} / \mathrm{dl}$, hypertensive if already on antihypertensive medication or recorded blood pressure of more than $140 / 90 \mathrm{mmHg}$ at three different occasions, smoker was defined as a person who smoked more than 100 cigarettes in his/her life time, obese if body mass index was more than $30 \mathrm{~kg} / \mathrm{m} 2$ and having dyslipidemia if total cholesterol level was more than $200 \mathrm{mg} / \mathrm{dl}$ or getting treatment with cholesterol lowering drugs. Positive family history for CAD in the patient was considered if the patient had a first-degree male relative of age less than 55 years or a first degree female relative of age less than 65 years had history of CAD. The data on demographic details such as age, gender, history of smoking, diabetes, hypertension and family history of ischemic heart disease was collected via a questionnaire. Blood samples were drawn for assessment of diabetes, dyslipidemia if not previously known. All patients also underwent ECG and cardiac specific trop-I assessment. Patients were categorized as NSTEMI with ST-elevation in aVR (yes/no) as per operational definition. Coronary angiography was performed in all patients as per hospital protocols. Coronary angiogram was reported by two senior consultant cardiologists with at least 5-year post fellowship experience by visual estimate and who were unaware of the ECG findings of the patients. Angiography findings were noted in pre designed Performa. All patients were managed as per hospital protocol.
The statistical Package for Social Sciences (SPSS) for Windows, version 23.0 was used for all analyses. Categorical variables like gender, dyslipidemia, hypertension, diabetes, smoking, stress, family history, ST-elevation in aVR lead and LMS disease and 3VCAD were presented as percentages and frequencies. Frequency of LMS and 3VCAD among patients with ST elevation positive in aVR and no ST elevation in aVR groups was compared by chi-square test. A p-value $<0.05$ was considered statistically significant. Data was stratified on patients age, gender, smoking, diabetes, hyperlipidemia, family history of ischemic heart disease to see the effects on frequency of left main and 3VCAD. Post stratification chi-square test was used. Specificity, sensitivity positive and negative predictive values of ST elevation in aVR for predicting LMS disease and 3VCAD was also calculated.

\section{RESULTS}

Total 346 patients were included in the study. Mean age of the patients was $51.87 \pm 10.03$ years. Minimum age was 30 years and maximum age was 70 years. There were $218(63.01 \%)$ males and $128(36.99 \%)$ females. Dyslipidemia was found in 141 (40.75\%), hypertension in $192(55.49 \%)$, diabetes in 168 $(48.55 \%)$ patients, $136(39.31 \%)$ were smokers and 76 $(21.97 \%)$ patients have family history of ischemic heart disease. Out of 346 patients, $182(52.60 \%)$ had ST elevation in aVR. Left main stem coronary artery disease was found in $85(24.57 \%)$ patients of NSTEMI. Triple vessel disease was found in 70 $(20.23 \%)$ patients of NSTEMI. Table 1 shows the comparison of LMS disease and 3VCAD in patients with and without ST-elevation in aVR. P-value was also mentioned in Table 1 along with specificity sensitivity, positive predictive values and negative predictive values. Table 2 shows the stratification of LMS disease and 3VCAD with ST elevation in aVR with different risk factors of CAD.

Table 1: Comparison of left main coronary artery and triple vessel disease among patients with and without ST elevation in aVR

\begin{tabular}{|l|c|c|}
\hline \multirow{2}{*}{} & \multicolumn{2}{|c|}{ ST elevation in aVR } \\
\cline { 2 - 3 } & Yes & No \\
\hline Left Main Stem Stenosis & $53(29.10 \%)$ & $32(24.60 \%)$ \\
\hline Yes & \multicolumn{2}{|c|}{0.038} \\
\hline No & \multicolumn{2}{|c|}{$62.35 \%$} \\
\hline P-value & \multicolumn{2}{|c|}{$50.57 \%$} \\
\hline Sensitivity & $29.12 \%$ \\
\hline Specificity & $80.49 \%$ \\
\hline Positive predictive value & \multicolumn{2}{|c|}{} \\
\hline Negative predictive value & $54(29.70 \%)$ & $16(9.80 \%)$ \\
\hline Triple Vessel Disease & $128(70.30 \%)$ & $148(90.20 \%)$ \\
\hline Yes & \multicolumn{2}{|c}{} \\
\hline No & \multicolumn{3}{|c|}{}
\end{tabular}




\begin{tabular}{|l|c|}
\cline { 2 - 2 } P-value & $<0.001$ \\
\hline Sensitivity & $77.14 \%$ \\
\hline Specificity & $53.62 \%$ \\
\hline Positive predictive value & $29.67 \%$ \\
\hline Negative predictive value & $90.24 \%$ \\
\hline
\end{tabular}

Table 2: Stratification of LMS disease and 3VCAD with ST elevation in aVR with different risk factors of CAD

\begin{tabular}{|c|c|c|c|}
\hline \multirow{2}{*}{ Risk factors } & \multicolumn{2}{|c|}{ ST elevation in aVR } & \multirow{2}{*}{ P-value } \\
\hline & Yes & No & \\
\hline & \multicolumn{2}{|c|}{ Left Main Stem Stenosis } & \\
\hline & Yes & No & \\
\hline Age $30-50$ years & 33 & 64 & 0.132 \\
\hline Age $50-70$ years & 20 & 65 & 0.166 \\
\hline Males & 31 & 84 & 0.041 \\
\hline Females & 22 & 45 & 0.414 \\
\hline Dyslipidemia & 26 & 47 & 0.009 \\
\hline Hypertension & 27 & 67 & 0.130 \\
\hline Diabetics & 26 & 67 & 0.105 \\
\hline Smokers & 20 & 51 & 0.497 \\
\hline \multirow[t]{3}{*}{ Family history } & 9 & 37 & 0.460 \\
\hline & \multicolumn{2}{|c|}{ Triple Vessel Disease } & \\
\hline & Yes & No & \\
\hline Age $30-50$ years & 25 & 72 & 0.018 \\
\hline Age $50-70$ years & 29 & 56 & 0.001 \\
\hline Males & 34 & 81 & 0.001 \\
\hline Females & 20 & 47 & 0.001 \\
\hline Dyslipidemia & 20 & 53 & 0.020 \\
\hline Hypertension & 30 & 64 & 0.001 \\
\hline Diabetics & 28 & 65 & 0.005 \\
\hline Smokers & 20 & 51 & 0.001 \\
\hline Family history & 14 & 32 & 0.080 \\
\hline
\end{tabular}

\section{DISCUSSION}

The simple and easy predictors of coronary complexities are always welcome by the cardiologists that can help in deciding treatment strategy. ${ }^{9}$ These predictors must be useful in making revascularization decisions as well as helpful for predicting adverse events after coronary angiography. ${ }^{10}$ ST-elevation in lead avR have been shown to be linked with LMS disease and 3VCAD. ${ }^{9-11}$

Hussien et al have shown that ST-segment elevation in lead aVR had good sensitivity $(77 \%)$ but moderate specificity $(65 \%)$, positive predicted value (PPV) of $64 \%$ and negative predicted value (NPV) of $78 \%$ in diagnosing LMS disease or 3VCAD and concluded that ST elevation in aVR could be used as a predictor before angiogram. ${ }^{12}$ Our study showed low sensitivity specificity and PPV of ST elevation in aVR for diagnosing LMS disease and 3VCAD while high negative predictive value for ST elevation in aVR for predicting LMS disease and 3VCAD. This could be due to the larger sample size in our study and division of studied population by Hussien et al in different groups according to severity of ST elevation in aVR.

Kosuge et al. in their study reported that ST-segment elevation in lead aVR as a good predictor of LMS stenosis based on ECG. The analysis of the study revealed that $78 \%$ sensitivity and $62 \%$ specificity of ST elevation in aVR for predicting LMS or 3VCAD. ${ }^{13}$ These results are comparable to our study Nabati et al showed that ST elevation in aVR is seen in $7.3 \%$ to $32.3 \%$ of the patients presenting with NSTEMI. ${ }^{14}$ Our study showed $52.60 \%$ patients have ST elevation in aVR. The difference in our study from Nabati et al is due to the criteria of labelling ST elevation in aVR. Nabati et al labelled ST elevation of more than $1 \mathrm{~mm}$ as ST elevation in $\mathrm{aVR}^{14}$ while we labelled ST elevation of more than $0.5 \mathrm{~mm}$ as ST elevation in aVR.

Iqbal et al conducted a study on patients with acute coronary syndromes having ST elevation in aVR and showed only 121 patients out of 249 of STEMI are true positives who have ST elevation in aVR and LMS disease while only 02 are true negative who do not have ST elevation in aVR and do not have LMS. In case of NSTEMI, 52 patients are true positive and 37 are truly negative. ${ }^{15}$ This difference from our study may be due to the difference in inclusion criteria in both studies. Iqbal et al have included patients with all types of acute coronary syndromes but we have included patients only with NSTEMI.

Our study also has limitations. This is a single center studied conducted on the patients admitted via emergency with limited number of patients. Therefore, this study doesn't represent the exact picture for the whole population of the country. So, it does not necessarily represent situations prevailing in other part of the country. So, the results may not be similar with large scale survey. The need of the hour is to conduct a larger survey with larger sample size at multiple cardiac centers of the country and this study could become the basis for that larger multicenter survey.

\section{CONCLUSION}

NSTEMI patients with ST elevation in aVR may have higher chances of having LMS disease or 3VCAD. There is high negative predictive value for ST elevation in aVR to predict LMS disease or 3VCAD.

\section{AUTHORS' CONTRIBUTION:}

HTU: Conceiving and designing the study, data collection, data analysis, formulation of result and writing the manuscript, and responsible for accuracy of results and integrity of research.

KAH MSS, AA: Conceiving and designing the study, interpretation of data, critical review of manuscript, approval of final draft, and responsible for accuracy of results and integrity of research.

Conflict of interest: Authors declared no conflict of interest. 


\section{REFERENCES}

1. Alzuhairi KS, Sogaard P, Ravkilde J, Azimi A, Maeng M, Jensen LO, et al. Long-term prognosis of patients with non-ST-segment elevation myocardial infarction according to coronary arteries atherosclerosis extent on coronary angiography: a historical cohort study. BMC Cardiovasc Disord. 2017;17:279.

2. Al-Khatib SM, Stevenson WG, Ackerman MJ, Bryant WJ, Callans DJ, Curtis AB, et al. 2017 AHA/ACC/HRS guideline for management of patients with ventricular arrhythmias and the prevention of sudden cardiac death: a report of the American college of cardiology/American heart association task force on clinical practice guidelines and the heart rhythm society. Circulation. 2018;138(13):272-391.

3. Ran P, Yang JQ, Li J, Li G, Wang Y, Qiu J, et al A risk score to predict in-hospital mortality in patients with acute coronary syndrome at early medical contact: results from the Improving Care for Cardiovascular Disease in China-Acute Coronary Syndrome (CCC-ACS) Project. Ann Trans Med. 2021;9(2):167.

4. Zhou M, Wang H, Zeng X, Peng Y, Zhu J, Chen Wet al. Mortality, morbidity, and risk factors in China and its provinces, 1990-2017: a systematic analysis for the Global Burden of Disease Study 2017. Lancet. 2019;394:1145-58.

5. Lindow T, Birnbaum Y, Nikus K, Maan A, Ekelund U, Palm O. Why complicate an important task? An orderly display of the limb leads in the 12-lead electrocardiogram and its implications for recognition of acute coronary syndrome. BMC Cardiovasc Disord. 2019;19(13):1-8.

6. Wang A, Singh V, Duan Y, Su X, Su H, Zhang M, et al. Prognostic implications of ST-segment elevation in lead aVR in patients with acute coronary syndrome: A meta-analysis. Ann Noninvasive Electrocardiol. 2021;26(1):e12811.
7. Derumeaux G, Ternacle J. Layer-specific strain in acute coronary syndrome: back to the future. Eur Heart J Cardiovasc Imaging. 2018;19(12):1325-6.

8. Cerit L. Chicken or the egg: ST elevation in lead aVR or SYNTAX score. Cardiovasc J Afr. 2018;28(2),100-3.

9. Yan AT, Yan RT, Kennelly BM, Anderson Jr FA, Budaj A, López-Sendón J, et al. Relationship of ST elevation in lead aVR with angiographic findings and outcome in non-ST elevation acute coronary syndromes. Am Heart J. 2007;154(1):71-8.

10. Neumann FJ, Sousa-Uva M, Ahlsson A, Alfonso F, Banning AP, Benedetto U, et al. 2018 ESC/EACTS Guidelines on myocardial revascularization. Eur Heart J. 2019; 40(2):87-165.

11. Hennings JR, Fesmire FM. A new electrocardiographic criteria for emergent reperfusion therapy. Am J Emerg Med. 2012;30(12):994-1000.

12. Hussien A, Battah A, Ashraf M, El-Deen TZ. Electrocardiography as a predictor of left main or three-vessel disease in patients with non-ST segment elevation acute coronary syndrome. Egypt Heart J. 2011;63:103-7.

13. Kosuge M, Kimura K, Ishikawa T, Ebina T, Shimizu T, Hibi K, et al. Predictors of left main or three-vessel disease in patients who have acute coronary syndromes with non-ST-segment elevation. Am J Cardiol. 2015;95(11):1366-9.

14. Nabati M, Emadi M, Mollaalipour M, Bagheri B, Nouraei M. STsegment elevation in lead aVR in the setting of acute coronary syndrome. Acta Cardiol. 2016;71(1):47-54.

15. Iqbal M, Iqbal MU, Munir U, Ali A, Irfan M, Maaz HM et al. Frequency of Left Main Coronary Artery Disease in Patients Presenting with Acute Coronary Syndrome Having ST Elevation in Lead aVR On Electrocardiogram. PJMHS. 2020;14(1):564-6.

\section{Address for Correspondence:}

Dr. Ammar Akhtar, Department of Cardiology CPEIC Multan, Ch. Pervaiz Elahi Institute of Cardiology (CPEIC), Multan, Pakistan, Pakistan.

Email: ammarakhtar176@gmail.com 